\title{
The effects of familiarity and orientation in the haptic change task
}

\author{
Morton A. Heller ${ }^{1} \cdot$ Nelson Adams $^{2} \cdot$ Jessie Shuemaker ${ }^{1} \cdot$ Toro Graven $^{3}$
}

Published online: 3 January 2020

(C) The Psychonomic Society, Inc. 2020

\begin{abstract}
In seven experiments we examined haptic memory for location, item information, and orientation for 12 tangible pictures, geometrical forms, and letter shapes in the change task. The study evaluated the influence of stimulus familiarity on change task performance. In the change task, participants are exposed to an array of patterns and subsequently have to indicate which ones have been altered on a second array. We also sought to determine whether prior demonstrations of female advantages in the haptic change task were reliable (Heller et al., 2010). The present experiments failed to replicate gender differences, overall with large samples. When participants were told about the nature of the picture change they should anticipate $\left(45^{\circ}\right.$ or $90^{\circ}$ rotation, location exchange), comparable performance was found for the groups in Experiment 1. In another experiment, participants were not told what sort of change might occur, and higher performance was found for the $90^{\circ}$ rotation group than for the location exchange and $45^{\circ}$ groups. Participants benefited from explicit instructions about the nature of the change. Telling participants the names of the raised-line pictures during initial study improved both change performance with the pictures and subsequent recognition. Changes derived from the substitution of new tangible print capital letters were easier to detect than location exchanges of the letters. Changes from letters to geometrical shapes were easy to detect. High performance resulted when participants were told to indicate which tangible letters were turned $\left(45^{\circ}\right.$ or $\left.90^{\circ}\right)$ or exchanged in a final experiment. The results suggest the importance of pattern familiarity in haptic spatial memory.
\end{abstract}

Keywords Haptics $\cdot$ Spatial memory $\cdot$ Touch

Relatively few studies have been conducted on detecting change in complex haptic 2-D arrays (see Gallace, Tan, \& Spence, 2006; Heller et al., 2010). However, Yoshida, Yamaguchi, Tsutsui, \& Wake (2015) reported extremely poor spatial memory for tactile change task performance compared with vision. Yoshida et al. studied orientation and location changes of vibrotactile straight lines, and did not examine spatial memory in the context of graphics and pictures for touch. Gallace et al. (2006) also used a vibrotactile array in their demonstration of tactile change blindness. The present experiments studied spatial memory in a haptic change task that used raised-line pictures and other graphical configurations involving letters and geometric shapes.

A much larger number of studies have examined change detection and change blindness in vision (see Auvrey, Gallace,

Morton A. Heller

maheller@eiu.edu

Eastern Illinois University, Charleston, IL, USA

Winston-Salem State University, Winston-Salem, NC, USA

3 University of Oxford, Oxford, UK
Tan, \& Spence, 2007; Luck, 2008; Pashler, 1988; Simons, 2000; Wood \& Simons, 2017). In one version of the visual change task, participants are shown an array comprised of a number of pictures for perhaps $1 \mathrm{~s}$, and are then shown a second array. The requirement is to indicate which pictures have been changed in the second array and which have not been changed (Silverman \& Eals, 1992). The changes could consist of a change in location, adding or substituting new patterns, or a change involving exchanging locations of some of the pictures (see James \& Kimura, 1997). In exchange methods, pairs of the pictures are interchanged (exchanged locations), but other pictures are not moved. In this case, no new pictures are added. A variety of paradigms have been used to study this problem in vision, including using photographs of complex natural scenes (but see Linn \& Luck, 2012; Luck, 2008; Silverman \& Eals, 1992).

The present study was also directed toward the consideration of the salience of spatial features in haptics, including orientation and it examined the impact of pattern familiarity. There is little doubt that vision is tuned to notice changes in orientation, and this is certainly a defining feature of many print letters. However, there has been some debate over whether the situation is identical for touch. For example, 
Millar $(1994,1997)$ has argued that orientation is not very salient for touch and this explains some of the difficulty in learning to read Braille. Many Braille symbols are defined by orientation, thus the difference between the character that represents a letter $\mathrm{B}$ and $\mathrm{a} \mathrm{C}$ is an orientation difference. The $\mathrm{B}$ is denoted by two vertical dots, and the $\mathrm{C}$ is comprised of two horizontal Braille dots. Also, Millar proposed that skilled blind readers of Braille tend to treat the symbols as a texture gradient, and do not normally process the shapes of the individual Braille characters. Thus, they may respond differently than novices or sighted individuals to stimulus rotation as an indicator of change.

Heller, Calcaterra, Green, and Lima (1999) examined the effect of orientation on the recognition of Braille characters. They found that left-right-reversed Braille was far easier for the blind participants than $180^{\circ}$ rotations. Blind participants are experienced with left-right-reversed Braille when they use portable Braille slates for writing. Rotations in the picture plane alter Braille character identity. This is a further reason for testing the ability of participants to notice rotation as compared with location changes, and also suggests the impact of pattern familiarity and experience.

It is possible that orientation shifts could alter the recognizability of many raised-line pictures. Alterations of picture orientation from canonical and familiar orientations, in vision or touch, could reduce the ease of picture identification. In addition, this could vary with the nature of the picture and the modality. Thus, the raised-line pictures in the experiments reported here were modifications of pictures that were visually normed, but we do not have comparable normative information for raised-line pictures using touch. Moreover, information is lacking on canonical orientations for raised-line pictures in haptics, as they are unfamiliar stimuli.

Earlier researchers in vision have reported an advantage for females in change detection (Eals \& Silverman, 1994; Silverman \& Eals, 1992). Eals and Silverman presented an array of 36 pictures for $1 \mathrm{~min}$ and then added new pictures to the array. Participants were asked to indicate which pictures were new and which ones were in the original array. In a further experiment, some of the pictures were moved to new locations, and the task was to indicate which ones were moved. Females did better than males when pictures were moved, but not when the task was to indicate which pictures were added. Silverman and Eals attempted to explain their results in terms of evolutionary theory.

More recently James and Kimura (1997) used an experimental method that was similar to that of Silverman and Eals (1992). In an exchange position (location exchange) condition, some of the pictures were interchanged in the second array. In another instance, some of the pictures were moved to completely new positions. James and Kimura found a small but reliable advantage for females in the exchange position condition, but not when the pictures were moved to new locations. They did not add new pictures in these manipulations. The investigation of sex differences in spatial reasoning and cognition shows that males tend to perform better than females in mental rotation problems (see Voyer, Nolan, \& Voyer, 2000; Voyer, Postma, Brake, \& Imperato-McGinley, 2007). However, in many cases females may perform at a comparable level to males in object location memory (see Postma, Izendoorn, \& De Haan, 1998), and they may even outperform males in the change task in vision, and perhaps in touch.

Heller et al. (2010) devised a haptic analogue of the visual exchange task used by James and Kimura (1997). Raised-line swell-paper pictures were used in place of visible line drawings for blindfolded individuals who were limited to haptic examination. The number of pictures was reduced considerably from the number that was used in visual experiments, since pilot data had indicated that blindfolded sighted participants were unable to cope with 22 tangible raised-line pictures in an array that was 11 in. $\times 17$ in. $(28 \mathrm{~cm} \times 43 \mathrm{~cm})$. Heller et al. (2010) reported an advantage for females in the haptic change task, but this advantage varied with task difficulty. When the task was more difficult, the superiority of females vanished.

It is important that larger raised-line pictures yield higher recognition performance (Kennedy \& Bai, 2002; Wijntjes, van Lienen, Verstijnen, \& Kappers, 2008). Also, the recognition of swell-paper pictures can be low, and studies have shown that it is often easier to recognize cutouts (Kalia et al., 2014). Kalia and Sinha (2011) reported large differences in the accuracy of recognition of raised-line pictures using tracing. Some raised-line pictures were very easy to recognize (e.g., the heart, close to ceiling rates of recognition), and others were very difficult (the hand was intermediate, and book close to floor), depending on the particular viewpoint and size of the picture.

The haptic change task was difficult for participants, and this raised a number of important issues. One might wonder whether haptic spatial perception and memory pose special problems that are apparently absent in vision and that may vary by modality (see Yoshida et al., 2015). Yoshida et al. argued that haptic spatial memory is severely limited and that this explains poor performance in tactile 2-D perception. Indeed, they suggested that "the haptic system is almost amnesic when operating outside of the fingertips" (p. 1209). Thus, one might expect that it could be difficult to recall and notice changes in an array of raised-line pictures, if one cannot tell what they are. Special difficulties could be involved in recalling haptic spatial information when pictures are involved. The present study was directed toward clarification of these issues. Consequently, many of the experiments compared rotation changes with location changes or changes in identity. These sorts of changes are different in important ways, but they allow an evaluation of the notion of spatial 
limitations in touch. Note that correct performance in the orientation change task with letters could derive from both noticing a change in the orientation of individual patterns and noticing deviations from the familiar, canonical orientation as the patterns were all initially upright in Experiments 5-7. Location shifts have been associated with change blindness (Gallace et al., 2006) and prompted very low performance in the study of orientation and location shifts by Yoshida et al. It was not known whether these sorts of outcomes would generalize to raised-line pictures and letters, or were limited to the simple vibrotactile straight line stimuli in the earlier study by Yoshida et al.

The overall aim of the experiments involving raised-line pictures was to evaluate the role of familiarity and item information in the haptic change task. Tangible pictures are relatively unfamiliar stimuli for blindfolded sighted and for blind individuals, and so it was thought that lack of familiarity might suppress performance. Further experiments were designed to provide converging evidence on the role of familiarity by using easily identifiable patterns, namely raised-line print capital letters and geometrical forms. Letters and simple geometrical shapes are more familiar than complex raised-line pictures, and large letters are easy to identify when one is restricted to touch (see Loomis, 1981). Letters are normally experienced in an upright orientation, and large deviations from canonical, familiar upright orientations can alter pattern identity and conceivably alter performance in the haptic change task. One would expect that the stimulus array should be relatively easy to remember when the stimuli are familiar letters. An underlying assumption of the study was that item information is potentially important for change task performance. Moreover, reports of changes in the letter array should generate higher accuracy levels than found with unfamiliar stimuli.

\section{Experiment 1: Lack of sex differences}

Experiment 1 compared the performance of males and females on the haptic change task. Given the results of earlier studies (e.g., Heller et al., 2010; James \& Kimura, 1997), females were expected to perform better than males. However, the advantage of females was not found throughout all of the experiments in Heller et al. (2010). It was probable that statistical power was limited in that earlier study, given a moderate sample size. Consequently, a larger number of participants were tested in this experiment $(N=120)$ compared with 30 and 32 in Experiments 1 and 2 in the previous study. If orientation is a special problem for observers using haptics, then one might expect higher change detection performance for the group that had location exchanges. One might also expect that it would be easier to notice larger orientation changes, so one would expect better performance in the $90^{\circ}$ than in the $45^{\circ}$ group. Obliques are more difficult to code and interpret in vision and in touch, and so we expected greater difficulties with $45^{\circ}$ rotations than $90^{\circ}$ rotations (Appelle, 1972; Gentaz, Baud-Bovy, \& Luyat, 2008).

\section{Method}

Participants There were 120 participants, with 40 (20 males and 20 females) in each of the three conditions: exchange, $45^{\circ}$ rotation, and $90^{\circ}$ rotation. All of these undergraduate volunteers (age range $=18-48$ years; five were over 22 ) were experimentally naïve to the aims of the experiment and were recruited from introductory psychology classes. The participants in the experiments reported here had normal or corrected-to-normal vision as indicated by self-report. All of the blindfolded sighted participants in the experiments in the present manuscript reported that they had normal touch.

Stimuli The stimuli were 12 swell-paper raised-line pictures on an 11 in. $\times 17$ in. $(28 \mathrm{~cm} \times 43 \mathrm{~cm})$ array. The pictures were simplified versions of the line drawings in Snodgrass and Vanderwart (1980). All of the pictures had high visual recall and name agreement scores. The individual pictures were all between $3.2 \mathrm{~cm}$ and $7 \mathrm{~cm}$ tall, with the exception of the 8.3$\mathrm{cm}$-long baseball bat. The pictures were all smaller than the hand and required fine finger and hand movements for exploration. The stimuli were in a relatively irregular arrangement, as in Experiment 2 of Heller et al. (2010). Figures 1, 2, 3, and 4 show the initial study array and the second test array, with some of the pictures moved to new locations (exchanged) or rotated $45^{\circ}$ or $90^{\circ}$ from their original positions. Note that the array remained horizontal and was fixed in place on the table surface, whereas the individual changed pictures were turned. The change in location was accomplished by exchanging three pictures with three others. The same pictures were selected for rotation as for exchange. Participants used square pieces of felt (12 mm square by $6 \mathrm{~mm}$ thick) to mark the

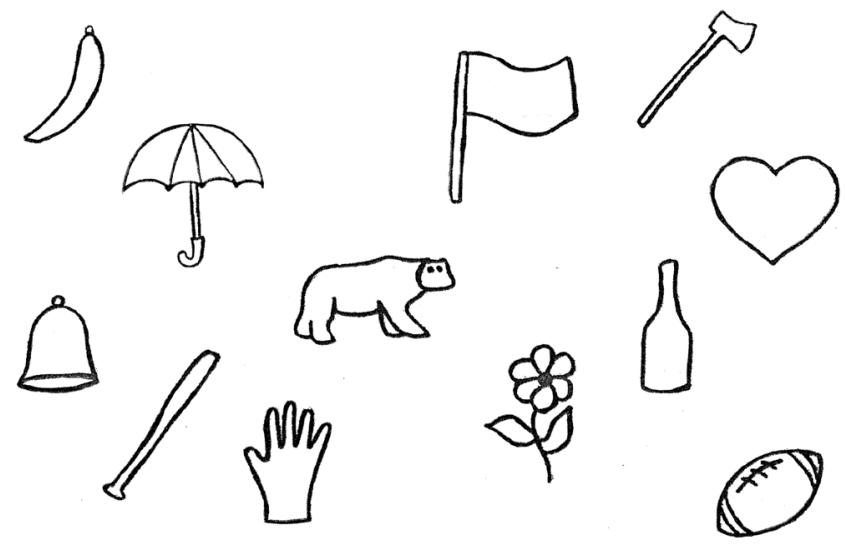

Fig. 1 The standard picture stimuli. The pictures are all smaller than hand size. 

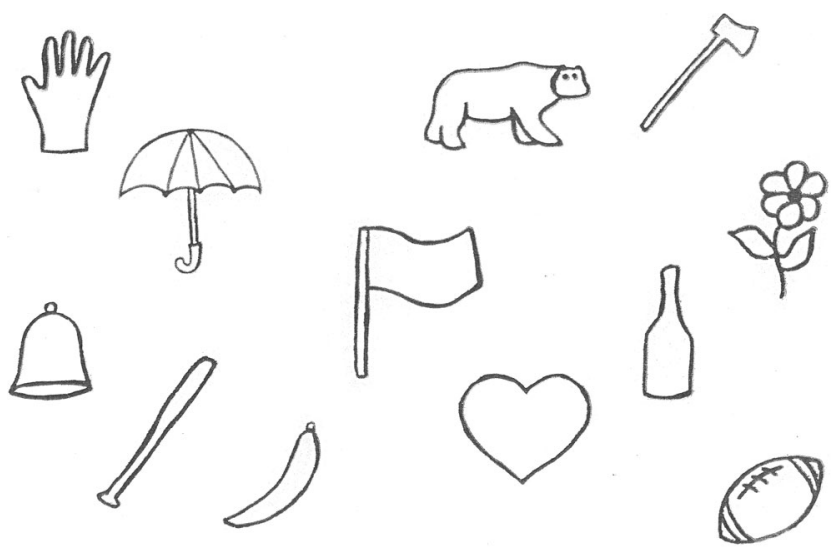

Fig. 2 Stimuli with location changes. Three of the pictures were exchanged with three others.

pictures they thought had been moved to new locations or turned. Figure 5 shows the experimental setup.

Design and procedure The experiment had a between-group factorial design with independent groups for gender (male vs. female) and type of change (location exchange vs. $45^{\circ}$ rotation vs. $90^{\circ}$ rotation). All of the participants were told about the layout of the framework that housed the picture array, and it was pointed out to them before blindfolding. Then the blindfolded participants were told that they should feel the tangible array to learn the locations of the pictures (or orientation), as appropriate. They were told that they would have $8 \mathrm{~min}$ to study the first array, and then they would be stopped. Subsequently, the first array was removed and a second array was placed at the same physical location, at the body midline. They were to indicate which pictures were moved to new locations, or which ones were turned from their original orientations. The time interval between the study and test sheets was brief and less than $10 \mathrm{~s}$. Participants were instructed that they could use one or both hands if they wished, and should be sure to use all of the $8 \mathrm{~min}$ for study. They were instructed to feel all of the pictures during that time. If they wanted to stop

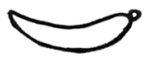

2
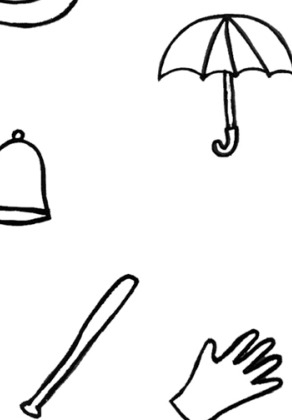
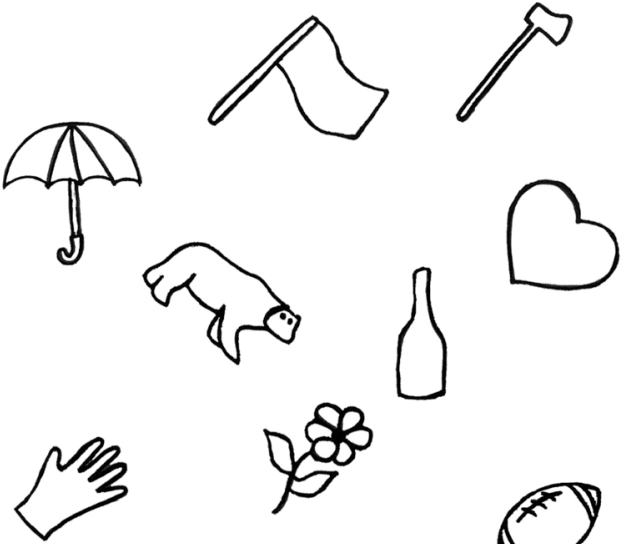

Fig. 3 Changes that entail $45^{\circ}$ rotations.
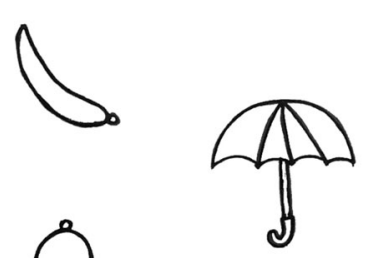

$\square$
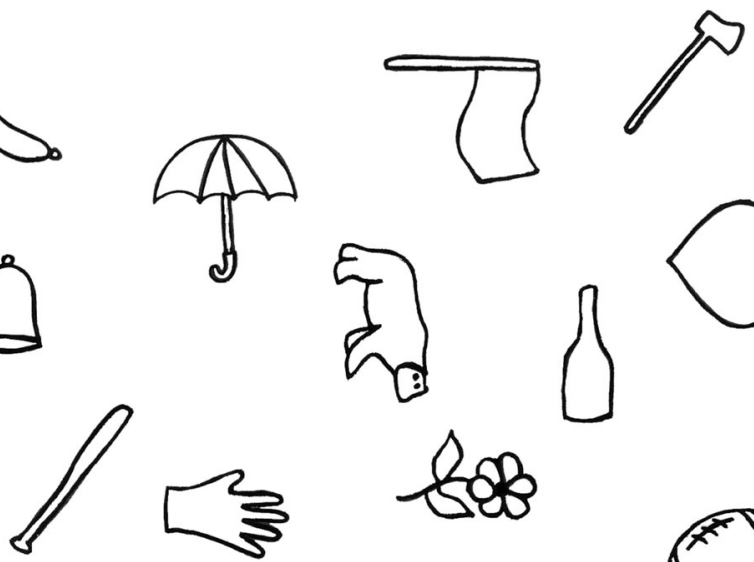

Fig. 4 Changes consisting of $90^{\circ}$ rotations.

before the 8-min study time was complete, they were told to continue studying the pictures. If by $4 \mathrm{~min}$ a participant had failed to feel all of the pictures, the individual was told "there could be some pictures that you missed." However, no further information was given; that is, participants were not guided to the pictures that had not been touched and studied.

Prior to initiation of the learning and testing session, a pile of square pieces of felt was placed at the left side of the tabletop. The blindfolded participants were verbally guided to this location and told to feel the pieces of felt. The participants were instructed to feel the second set of stimuli and to indicate which ones were changed by placement of the squares on those patterns that had changed. In addition, they were instructed that they would be timed at test, but that they should try for accuracy. We provided accuracy instructions since we were concerned that attempts at speed would lead to low scores and perhaps instances of participants failing to feel all of the pictures. Feedback on performance was not given. They were told that the pictures were of things that could be named,

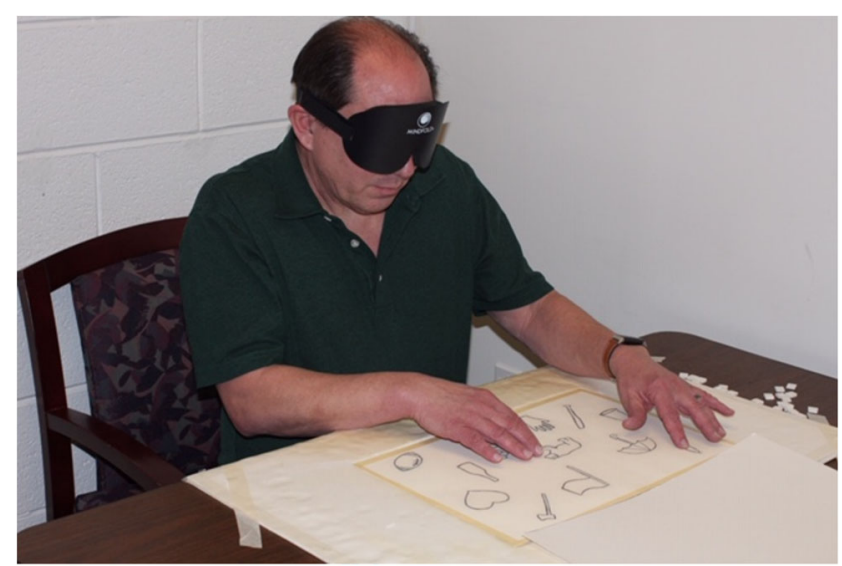

Fig. 5 The experimental arrangement. The swell-paper stimuli were placed within a foam board frame. The frame was fixed in place by tape, ensuring that the orientation of the entire array did not vary. The photograph has N.A. seated with the standard study array at the midline, in the position of the participants. 
if they wanted to do so. If participants asked if they should name the pictures, they were told that "now, the task is to try to learn the locations (or orientation) of the pictures." The participants were told that their score would derive from correctly indicating which pictures were changed and from correctly indicating which pictures were not changed.

After completion of the change task, the participants were asked to feel the tangible pictures again, while still blindfolded, and try to name them. They subsequently attempted this using sight. Almost all of the participants correctly named all of the pictures using sight, with a few exceptions. One person said that she did not know what to call the visible picture of the axe, but later (incorrectly) called it a hammer. Another participant called the axe a machete. The bear was called a hippo by one person, a cow by two others, and another individual said it was an animal, but didn't know what to call it.

\section{Results and discussion}

The results are shown in Table 1 and indicate similar mean numbers of correct change scores for the $90^{\circ}$ change group ( $M$ $=9.1$ correct, $M=76 \%$ correct) as for the exchange position $(M=8.4$ correct, $70 \%)$ and $45^{\circ}$ rotation $(M=8.8$ correct, $73 \%)$ groups. An analysis of variance (ANOVA) on mean numbers correct showed that the effect of group failed to reach significance, $F(2,114)=1.14, p=.32$. Males appeared to do better than females given the $90^{\circ}$ rotation, but the effect of gender failed to reach significance, $F(1,114)=1.6, p=.21$, and the interaction was nonsignificant $(F<1)$.

Mean solution times were slightly longer for the $45^{\circ}$ group $(M=316.5 \mathrm{~s})$ than for the $90^{\circ}$ group $(M=247.8 \mathrm{~s})$ and the exchange position group $(M=271.9 \mathrm{~s})$. However, the effect of group on solution times was nonsignificant, $F(2,114)=3.0, p$ $=.054, \eta_{\mathrm{p}}^{2}=.0498$.

Table 1 Mean numbers correct for the haptic change task ( $S D \mathrm{~s}$ in parentheses), mean solution times (in seconds), and haptic picture identification mean numbers correct for Experiment 1, with participants told that some raised-line pictures have been moved to new positions or turned

\begin{tabular}{llll}
\hline Change Group & Change & Time & Identification Out of 12 \\
\hline \multicolumn{2}{l}{ Exchange Position } & & \\
Male & $8.55(2.1)$ & $235.15(104.1)$ & $2.75(1.5)$ \\
Female & $8.15(2.9)$ & $308.55(129.4)$ & $3.50(2.3)$ \\
$45^{\circ}$ Change & & & \\
Male & $8.85(1.9)$ & $302.40(143.6)$ & $3.70(2.7)$ \\
Female & $8.70(1.9)$ & $330.55(170.0)$ & $4.25(2.6)$ \\
$90^{\circ}$ Change & & & \\
Male & $9.60(2.0)$ & $256.55(106.0)$ & $3.90(2.3)$ \\
Female & $8.60(2.5)$ & $239.25(95.1)$ & $3.25(2.8)$ \\
\hline
\end{tabular}

Total $N=120 ;$ maximum score possible $=12$ correct.
Performance in the picture identification task, undertaken after the change task, was poor, with mean recognition scores ranging from $23 \%$ correct to $35 \%$ correct (see Table 1). An ANOVA on the number of pictures correctly identified by touch showed that all of the main effects and interactions failed to reach significance (all $p \mathrm{~s}>$.29). The identification task was used to rule out the possibility that if there were effects of group on the change task, they were not the result of orientation altering haptic picture identification. Conceivably, variations in picture orientation could have altered identification accuracy for pictures that were rotated. Note that visual identification of the pictures was virtually errorless. Haptic identification performance with raised-line pictures depends on stimulus size, and lower performance has been found with smaller patterns (see Kennedy \& Bai, 2002; Wijntjes et al., 2008). For example, Wijntjes et al. found lower recognition performance with 10 -cm stimuli $(77 \%$ correct) than with $30-\mathrm{cm}$ (84\% correct) swell-paper pictures. Kalia and Sinha (2011) used swell-paper drawings within a space that was $11 \mathrm{~cm}$ high and $16 \mathrm{~cm}$ wide. Kennedy and Bai (2002) reported mean recognition scores of $61 \%$ correct for large pictures, whereas much lower performance was reported by Heller (1989) with relatively small pictures. It is important that the individual pictures differed across these studies, along with stimulus size and method of production (see Wijntjes et al., 2008).

The results of Experiment 1 indicate that if told that they should expect orientation changes, participants are certainly able to notice these changes and point them out. Note that the first experiment included orientation changes and changes in location. An anonymous reviewer suggested some caution in the interpretation of the outcome, since some orientation changes could be easier to notice than changes in location, and vice versa. If gender were a problem for particular types of stimuli, this caution might be less significant. In other instances, it might be more important. Moreover, gender did not matter, given larger samples $(N=120)$ than in the experiments in a previous study ( $N \mathrm{~s}$ of 30 or 32 ). However, it was not known whether orientation would be salient for touch without this instruction. This question was tested in Experiment 2.

\section{Experiment 2: Rotation versus location exchange without prior information about the nature of the change}

The results of Experiment 1 showed that participants could recall the positions and orientations of the pictures in an array using touch when they were informed about the nature of the change. However, it was expected that orientation would be less salient without prior information about the change. In this experiment, the participants were merely told that they were to indicate if any of the pictures were changed in any way, but 
were not informed about the precise change they should anticipate. It was expected that the $45^{\circ}$ rotation group should find the task more difficult than the $90^{\circ}$ rotation group, given demonstrations of the oblique effect in vision and touch (see Gentaz \& Hatwell, 1995).

\section{Method}

Participants There were 12 males and 12 females in each of the three change manipulations, for a total $N$ of 72 . Their ages ranged from 18 to 44 years, with five participants over the age of 22. None of the participants served in Experiment 1.

Stimuli and apparatus The stimuli were identical to those of the first experiment.

Design and procedure The experiment had an independentgroup $2 \times 3$ factorial design, with gender (male vs. female) and type of change (location exchange vs. $45^{\circ}$ rotation vs. $90^{\circ}$ rotation) as the factors. In all respects other than the instructions, the procedure was the same as in Experiment 1. The participants were told that there would be changes in some of the pictures on the second sheet, but they were not told anything else about these changes. They were told that some of the pictures would be "changed," but also that some would not be changed.

\section{Results and discussion}

The results of Experiment 2 are shown in Table 2 and indicate a higher mean number correct for the $90^{\circ}$ rotation, as well as slightly better performance by females $(M$ correct $=8.0$ ) than among males $(M$ correct $=7.3)$. In order, the mean numbers correct for the $45^{\circ}$, exchange position, and $90^{\circ}$ groups were 7.0, 7.4, and 8.7, respectively. An ANOVA on numbers correct showed that the effect of type of change was significant,

Table 2 Mean numbers correct for the haptic change task and mean solution times (in seconds) for Experiment 2, with participants told that "some pictures have been changed"

\begin{tabular}{lll}
\hline Group & Correct Out of 12 & Time Score (Seconds) \\
\hline $\begin{array}{lll}\text { Exchange Position } \\
\text { Male }\end{array}$ & \\
Female & $7.0(1.5)$ & $303.58(158.8)$ \\
$45^{\circ}$ Change & & $276.58(104.9)$ \\
Male & $6.50(1.5)$ & \\
Female & $7.42(1.7)$ & $355.00(112.0)$ \\
$90^{\circ}$ Change & & $298.08(139.9)$ \\
Male & $8.50(1.6)$ & $325.33(100.4)$ \\
Female & $8.92(2.3)$ & $297.83(139.9)$ \\
\hline
\end{tabular}

Maximum score possible $=12$.
$F(2,66)=6.55, p=.003, \eta_{\mathrm{p}}{ }^{2}=.166$. A Newman-Keuls test on the means indicated that the $90^{\circ}$ rotation mean number correct was significantly higher than the location exchange and $45^{\circ}$ means $(p<.05)$, which did not differ from each other $(p>.05)$. The interaction between type of change and gender was nonsignificant, $F<1$. A separate ANOVA was conducted on response latencies, and none of the main effects or interactions reached significance (all $p \mathrm{~s}>.21$ ).

The accuracy results of Experiments 1 and 2 were reanalyzed and considered as one experiment with a factorial design, to examine the effect of instructions. This independent-group ANOVA had two instructions (explicit instruction about the change vs. told "changed") by two genders (male vs. female) by three changes (location exchange vs. $45^{\circ}$ vs. $90^{\circ}$ ) as factors. The effect of instructions was highly significant, with higher mean numbers correct for participants given explicit instructions about the nature of the change $(M$ $=8.7,72.5 \%)$ than for those who were just told to indicate pictures that were changed $(M=7.7,64.2 \%), F(1,180)=11.9$, $p=.001, \eta_{\mathrm{p}}^{2}=.062$. Overall, we found a significant effect of type of change, owing to higher performance in the $90^{\circ}$ group $(M=8.9)$ than in the $45^{\circ}(M=7.9)$ and exchange position $(M$ $=7.9)$ groups, $F(2,180)=5.1, p<.01, \eta_{\mathrm{p}}{ }^{2}=.053$. The effect of gender failed to reach significance $(F<1)$, and the interaction between gender and instruction was nonsignificant, $F(1$, $180)=3.87, p=.051$. This nonsignificant interaction indicates that any conclusions about gender differences are still very questionable. The lack of a significant female advantage over males in the task suggests that prior reports of gender differences in haptics are not reliable (Heller et al., 2010).

The results showed that $45^{\circ}$ rotations were more difficult to notice than $90^{\circ}$ rotations of pictures. Accuracy scores were also lower than when the participants in Experiment 1 were given explicit instructions to look for pictures that might be turned. Future work in this area might inform participants about the number of pictures that have changed, as this could be a more sensitive measure of change detection. Note that although the pictures were not presented within a broader context, the foam board frame and mat mounting board did provide a spatial frame of reference. The framework thus permitted information about the horizontal and vertical, if the context was felt by participants.

Task difficulty can be high in the haptic picture change task when instructions are not explicit and there are a number of conceivable causes. One possible explanation is that the lack of experience and familiarity with particular haptic graphic patterns may contribute to this difficulty. This prompted further investigation and was examined in the subsequent experiments. Note that performance in the haptic task can be similar to that in the visual version, but people need more study time even when examining relatively small numbers of haptic pictures. Also, it is important to recognize that the experiments compared different types of spatial transformations, and 
caution should be exercised in the interpretation of the results. An anonymous reviewer pointed out that the benefit of explicit instructions only produced a modest gain in performance from $64.2 \%$ to $72.5 \%$. This may suggest that the changes were relatively noticeable to a number of people using touch.

Therefore, an experiment was conducted to provide further clarification of whether the difficulty found with the haptic change task and noticing rotation derived from haptics per se or from difficulty of identification of the tangible pictures (see Exp. 1). This follow-up visual experiment replicated the methods of Experiment 2, and 13 individuals (two males) used sight for the picture change task, in which the change involved $45^{\circ}$ rotations (age range $=19-23$ ). An additional 12 female participants used vision with $90^{\circ}$ changes (age range $=$ 19-22 years). Higher performance was expected in the $90^{\circ}$ change group. None of these participants had served in Experiment 1 or 2 . The initial exposure time was $1 \mathrm{~min}$. In almost all other respects, the method was identical to that of Experiment 2, in which no prior information was given about the nature of the change. Performance was significantly better with $90^{\circ}$ rotations $(M=11.3, S D=0.98, M \%$ correct $=94)$ than with $45^{\circ}$ rotations $(M=10.5, S D=0.97, M \%$ correct $=$ $87.8), t(23)=2.04, p<.05$, one-tailed. A one-tailed test was used, since the second experiment with touch suggested increased difficulty with $45^{\circ}$ changes, in the absence of explicit instructions about rotations. Higher mean scores were predicted for the $90^{\circ}$ group, prior to data collection. In addition, response time was slightly shorter with $90^{\circ}$ rotations $(M=$ $31.3 \mathrm{~s}, S D=16.9)$ than $45^{\circ}$ rotations $(M=43.3 \mathrm{~s}, S D=$ 19.0), but the effect failed to reach significance, $t(23)=1.65$, $p>.05$. Performance using vision was higher than we had found in touch. These results suggest the possibility that people have little difficulty visually noticing some types of changes in arrays of pictures, even if they are not given prior information about the nature of the change. However, this is not the whole story.

There are problems with the assumption that the difficulties in the change task lie solely with haptics; it is possible that rapid and automatic visual identification of many pictures may have suggested canonical and plausible orientations to the observers for the first picture array in studies like James and Kimura (1997). Thus, for example, we would expect to see the picture of the bear walking on all four legs in an upright orientation (as in Fig. 1), and this was changed with the second set of pictures in the present study. Indeed, the original set of pictures included many of the changed pictures initially in an upright orientation. The higher visual performance might have been prompted by this increased familiarity with visual versus haptic pictures. Consequently, if participants saw a picture of a bear tilted $90^{\circ}$, this would be most obvious, since they might expect it to be upright, or perhaps turned at a smaller angle. Note that the pictures derived from a visually normed set (Snodrass \& Vanderwart, 1980), and we do not have comparable haptic norms for picture recognition. All of the visually normed pictures had high name agreement and recall scores in Snodgrass and Vanderwart's study.

To test this possibility, ten new participants (age range $=$ $18-22$ years) were visually tested with the second set of test pictures as the standard, and the changed pictures were presented first for testing with no prior information about the nature of the change. For example, the picture of the bear was tilted $45^{\circ}$ in what now became the first set of standard study pictures (Fig. 3). The picture of the hand was tilted in the first set of pictures but upright in the second set, and so forth. This method used the pictures from Experiments 1 and 2 (Fig. 3 ), but presented the pictures with some tilted first, and the other set second (Fig. 1). Thus, the test set became the standard picture set. The visual task was far more difficult using this method, with the mean number correct being considerably lower $(M$ number correct $=8.1 ; M \%$ correct $=67.5)$ than with vision in the forward sequence $(M$ number correct $=10.5)$. The difference between the means was significant, $t(21)=$ $4.55, p<.001$. Here, visual performance with a reversed sequence was not all that different from that obtained using touch. These results suggest that the difficulty found in the earlier experiments was, in part, a function of a lack of familiarity with haptic pictures, and not solely with limitations in haptics.

The results suggest that change task difficulty might be influenced by stimulus familiarity. Participants using touch are not familiar with the pictures that they touched, and had difficulty naming them. One consequence of this is that one can't readily identify canonical or altered orientations with unfamiliar tangible pictures. Vision may benefit from knowledge derived from familiarity with canonical orientations. This can certainly have an impact on performance in both modalities.

It is important to mention that it is questionable to attempt simple and direct comparisons between vision and touch, especially when there are differences in stimulus familiarity. It certainly matters whether it is easy to identify selected pictures using vision, but not so readily using touch. Moreover, it is also very difficult to properly equate exposure time when comparing performance across modalities. These concerns suggest caution when attempting modality comparisons.

\section{Experiment 3: Effect of knowledge of picture identity}

The pictures used in Experiments 1 and 2 were visually normed, and visual identification was relatively easy. However, it is not as easy to name these small tangible pictures using touch (see Kennedy \& Bai, 2002; Wijntjes et al., 2008), perhaps due to lack of familiarity with using touch for picture perception. If one does not know the name or identity 
of a picture that is felt while blindfolded, it is probably more difficult, but not impossible, to use verbal coding. A lack of knowledge of the raised-line pictures should lower performance in the haptic change task.

Perhaps the difficulties with recognition of raised-line pictures can be related to limitations in tactile scanning. Kalia et al. (2014) reported higher levels of haptic picture recognition overall with cutouts (approximately $60 \%$ correct) compared with $40 \%$ correct for swell-paper pictures (Kalia \& Sinha, 2011). Kalia et al. suggested that blindfolded sighted individuals are hampered when exploring 2-D tangible pictures, since they can't use many of the exploration methods that are available when feeling solid objects. Thus, it is easy to use contour following when feeling a raised-line picture, but not enclosure. They argued that this can impair perception of global shape. This limitation in available exploration methods is likely a contributing factor to lower performance levels when attempting naming of tactile pictures, but performance levels also vary with the picture. Kalia and Sinha reported recognition levels that were higher than $60 \%$ for some tangible pictures, including a butterfly, kite, banana, hanger, arrow, moon, and heart. Perhaps constrained exploration methods contribute to difficulties with identification of raised-line patterns that prompt unfamiliar exploration methods and also contain fine details that are beyond the sensitivity of the fingertips or may be missed by use of a single exploration method. The reduced sensitivity problem, as compared with vision, is consistent with improvements in haptic picture identification with increased pattern size (Kennedy \& Bai, 2002; Wijntjes et al., 2008). This speculation is consistent with the overall logic of the Kalia et al. idea about difficulties with getting information about global shape with some raised-line patterns. Also, it offers another possible source of difficulty with tracing small raised-line pictures.

Heller (1989) found that providing blind, low-vision, and sighted participants with the set of names of 12 raised-line pictures aided later identification. However, the labels were never paired with individual pictures in that earlier study. Heller examined the effect of visual experience on picture naming but did not test for an effect of knowledge of picture labels on memory of a complex spatial array. In Experiment 3 we tested the effect of providing the names of the pictures while participants first began to explore them during the 8min study phase. It was expected that change task performance would be aided by telling the participants what the depictions represented when they first began to feel them during the initial study period. A control group was not given this information.

\section{Method}

Participants There were two independent groups with 11 in each, total $N=22$; age range $=18-23$ years. The group that was given the names of the pictures as they first explored them had two males and nine females. There was one male in the control group. The undergraduate participants had not been included in any of the prior experiments. All had normal or corrected-to-normal vision. They were recruited from an introductory psychology participant pool.

Stimuli The stimuli included the same 12 swell-paper tangible pictures used in Experiments 1 and 2, except that they were arranged in three horizontal lines, with four pictures in each line. Thus, the banana, umbrella, flag and ax were in the first, top row, the bell, bear, bottle and heart were in the second horizontal row, and the baseball bat, hand, flower and football were in the bottom row. Participants were not told anything about the spatial arrangement. Horizontal rows were adopted since this may facilitate the use of verbal coding (see Heller et al., 2010), and maximize the effect of naming. The spatial change for both groups involved exchanging three pictures with three others, exactly as in Experiments 1 and 2.

Design and procedure There were two independent groups, with one group given the name of each picture as participants began to feel them, and the second group denied this assistance. In most other respects, the method was like that of Experiment 2. The participants were told that they had $8 \mathrm{~min}$ to study the pictures, and that after this time a second set of pictures would be substituted. Here, they were to indicate which pictures were changed in any way. As they felt the pictures a second time during the study period, they were allowed to ask what they were if they forgot the name. As in earlier experiments, they were told that they would be timed, but should try for accuracy. Upon completion of the change task test, the participants were all asked to feel the tangible pictures and try to name them. This was done to ensure that the experimental manipulation was effective and aided knowledge of picture identity. Conceivably, we could have named the pictures for the participants but they could have forgotten what they were told. They were not told picture names during the testing phase of the change task or during the subsequent identification.

\section{Results and discussion}

Change task performance was significantly higher when the pictures were named for the participants $(M$ number correct $=9.6$ ) during the study period, $t(20)=2.07, p<$ .05 , one-tailed. Control group performance was clearly lower $(M=7.7$, see Table 3$)$. A one-tailed test was used, since a directional prediction was made prior to data collection. Prior to data collection, the group given picture names was hypothesized to outperform the control group in the change task. The mean number correct in the present control group was very similar to the scores in the no- 
Table 3 Mean numbers correct in Experiment 3, mean solution times (with $S D$ s in parentheses), and mean numbers of correct picture identifications for the haptic picture exchange position task, for participants told the names of the pictures versus those who were not told

\begin{tabular}{llll}
\hline Group & $\begin{array}{l}\text { Correct } \\
\text { Change }\end{array}$ & $\begin{array}{l}\text { Time Score } \\
\text { (Seconds) }\end{array}$ & $\begin{array}{l}\text { Haptic Picture } \\
\text { Identification }\end{array}$ \\
\hline Told picture names & $9.6(1.9)$ & $347.3(152.0)$ & $9.3(2.5)$ \\
Not told names & $7.7(2.2)$ & $283.1(113.3)$ & $3.6(2.0)$ \\
\hline
\end{tabular}

The participants were told that something would be changed in some of the pictures. Maximum score possible $=12$.

information exchange position group in Experiment 2 (see Table 2). Thus, it probably did not matter whether the pictures were arrayed in horizontal rows or an "irregular" arrangement. A $t$ test on response latency failed to reach significance, $t(20)=1.1, p=.27$. However, upon completion of the change task, vocal haptic identification of the raised-line pictures was significantly higher in the initialnaming group, $t(20)=5.9, p<.0001$ (see Table 3 ).

Naming the pictures for the participants during the study phase helped them in the change task and also helped them identify the pictures upon completion of the change task $(M=$ $78 \%$ correct). The mean number correctly identified was considerably lower in the control group $(M=3.6)$, which was nearly identical to the accuracy for picture identification in Experiment 1. Recognition performance in the present control group was higher than that reported by Heller (1989) for sighted controls before being given the set of picture labels. However, the pictures were different in the earlier study, and names were never provided for the individual pictures in that earlier study. Note that visual recognition was slightly less than $100 \%$ in Experiment 1, and perhaps would be $100 \%$ with some slight variations in the depictions. Recall that the drawings in the present experiments were modifications of those in the Snodgrass and Vanderwart (1980) study.

\section{Experiment 4: Orientation versus spatial location exchange in blind participants}

This experiment compared the performance of blind participants on an orientation change task, versus one in which the change involved spatial location. If increased experience with tactile form perception confers an advantage in spatial memory for blind individuals, one would expect them to show higher levels of performance in this experiment, even for the $45^{\circ}$ rotation condition. Although blind persons may be relatively unfamiliar with tangible raised-line pictures, groups of late-blind have shown better picture recognition than blindfolded sighted persons in earlier research, and some early-blind individuals have also performed better than the sighted (e.g., Heller, 1989). Some individuals blinded in very early childhood from retinoblastoma have exceptional spatial skills.

\section{Method}

Participants The visually impaired participants $(M$ age $=44$ years) were 11 totally blind individuals (ten late blind) recruited in Charleston, Champaign, LeRoy, Bloomington, and the Danville area of central Illinois. None of the participants had any significant pattern perception, but some had minimal light perception (see Table 4). Note that diabetic retinopathy is a very common cause of blindness, and diabetics frequently suffer from reduced tactile sensitivity. The 11 sighted participants were recruited from the students, faculty, and staff at Eastern Illinois University (age range $=19-62$ years, $M$ age $=42$, four males).

Stimuli and apparatus There were two conditions, picture location change and a change involving rotations of some of the pictures of $45^{\circ}$. Since each participant was exposed to both conditions, it was necessary to produce a second set of pictures, in addition to those from the earlier experiments, so that different picture sets were used for the location change and $45^{\circ}$ rotation conditions for individual participants (see Fig. 6). This permitted control over picture set difficulty. The swellpaper picture stimuli in the second set were similar in size to those of Experiments 1-3, but the smallest was $2 \mathrm{~cm}$ high by $5 \mathrm{~cm}$ wide (lips). The carrot was $8 \mathrm{~cm}$ long. The pictures in the second set of stimuli were a frying pan, envelope, airplane, hammer, necktie, guitar, butterfly, lips, carrot, key, turtle, and boot.

Design and procedure The experiment repeated measures on the type of change task, location change versus rotation $\left(45^{\circ}\right)$, with visual status as a between-group variable. About half of the participants started with the location change task, and half started with the orientation change task. For about half the participants, the location task used one set of pictures, and for the other half of the participants, the second set of pictures was used. The same method was followed for the $45^{\circ}$ orientation shift. This ensured that controls were applied for possible sequence or practice effects, as well as controlling for picture difficulty. As in Experiment 1, the participants were told, as appropriate, that they should try to identify the stimuli that were moved to new locations or the pictures that were turned $\left(45^{\circ}\right)$.

In most respects, the method was like that of Experiment 1 . However, the exposure/study time was reduced to a maximum of 6 min, since pilot work with a blind individual suggested that some blind people might find the 8-min study time too long. The aim was also to reduce the overall time for the experiment. Participants were allowed to end the study period when they thought 
Table 4 Characteristics of the blind participants in Experiment 4, including age, education, cause of blindness, fluency in Braille (BF), and the presence of light perception (LP)

\begin{tabular}{|c|c|c|c|c|c|}
\hline Sex & Age & LP & Cause & Education & $\mathrm{BF}$ \\
\hline $\mathrm{M}$ & 65 & No & $\begin{array}{l}\text { Virus, Glaucoma, retinal } \\
\text { detachment (42) }\end{array}$ & Ph.D. & Yes \\
\hline M & 42 & No & Hydrocephalus (9.5) & MA & Yes \\
\hline $\mathrm{F}$ & 49 & Minimal & ROP (39) & MA & Yes \\
\hline $\mathrm{F}$ & 48 & No & Retinal detachment (21) & $\mathrm{BA}$ & Yes \\
\hline M & 24 & No & Glaucoma, corneal loss (5) & HS & Yes \\
\hline $\mathrm{F}$ & 22 & No & Retinal detachment (12) & $\mathrm{SC}$ & Yes \\
\hline $\mathrm{F}$ & 35 & Some & $\begin{array}{r}\text { Nystagmus, retinal } \\
\text { deterioration ( } 8)\end{array}$ & $\mathrm{SC}$ & No \\
\hline $\mathrm{F}$ & 69 & No & Meningitis (2) & HS & Yes \\
\hline M & 38 & Yes & Diabetic retinopathy (35) & MA & Yes \\
\hline $\mathrm{F}$ & 58 & & Retinal detachment (15) & SG & No \\
\hline $\mathrm{F}$ & 34 & No & ROP (Birth) & $\mathrm{SC}$ & Yes \\
\hline
\end{tabular}

HS refers to a high school degree, SC means some college, MA indicates a master of arts graduate degree, and SG indicates some graduate school courses. The age of onset of blindness is indicated by a numeral in parentheses. ROP refers to retinopathy of prematurity.

that they knew the array and had sufficient study to allow them to tackle the change task. We opted not to ask for picture recognition, since it was thought that this might slow down the participants (Heller, 1989). Given the use of a repeated measures design, this would have been problematic for practical reasons, as it would have increased the overall time for the experiment. This was not pragmatic, as many of the participants who were visually impaired were tested at a workplace, Horizons for the Blind in Crystal Lake, Illinois. It was also thought that this design could present a difficulty for the blindfolded sighted participants, as it would make for a long test session and generate fatigue for both groups.

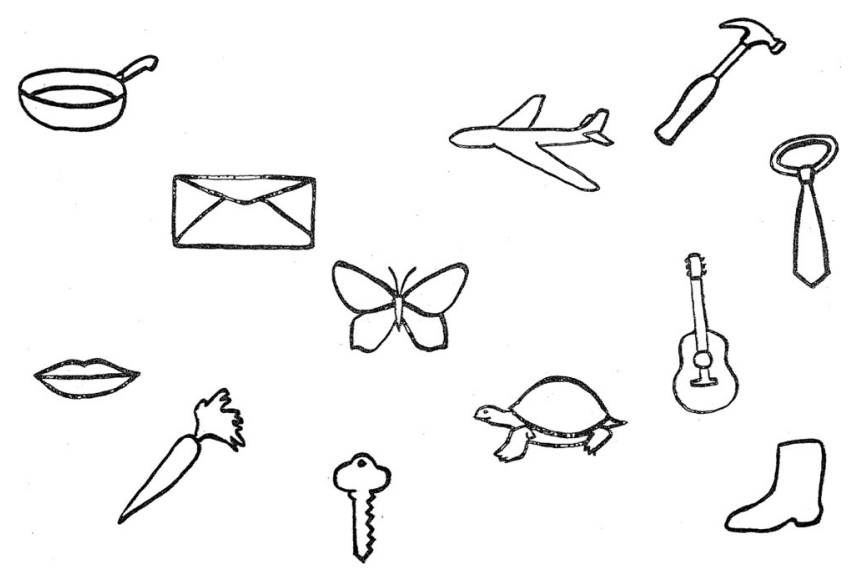

Fig. 6 The second set of standard pictures used in Experiment 4, in addition to the pictures shown in Fig. 1. The change conditions included $45^{\circ}$ rotation and exchange position.

\section{Results and discussion}

Performance in terms of numbers correct was similar for the orientation and location change conditions. Similar performance was also found for the blindfolded sighted and the blind participants (see Table 5). An ANOVA on numbers correct indicated that the effect of group failed to reach significance $(F<1)$, and the main effect of rotation and the interaction between group and task (rotation or exchange) also failed to reach significance (both $F \mathrm{~s}<1$ ). The blind participants appeared to be faster than the blindfolded sighted participants in the amount of time they required for study, but the effect of visual status failed to reach significance $F(1,20)=3.9, p=$ .06. A second ANOVA on response times also failed to reach significance $(F<1)$.

Overall performance was similar and only slightly lower than that found in the first experiment, despite a few differences in method and design. The present experiment repeated measures on type of change. In addition, the initial study time was reduced to a maximum of $6 \mathrm{~min}$, but the mean study time was less than $5 \mathrm{~min}$ for the blind participants. Furthermore, the second set of pictures was new. Nonetheless, similar performance levels were found for exchange position conditions in Experiments 1 and 4. Somewhat lower performance was obtained for the $45^{\circ}$ rotation change condition than in the results for blindfolded sighted individuals in Experiment 1. Perhaps that manipulation is more susceptible to the effects of reduced study time. However, it is also possible that the difference in the ages of participants could also explain this small difference, or that the minimal difference was due to chance.

Although one might expect higher levels of performance in the blind group than in the sighted, this was only hinted at for the response measure of initial study time and did not appear in accuracy. The effect of group on exposure/study time was nonsignificant. Also, the prior experience of the blindfolded sighted participants with many of the pictures in their daily lives did not seem to alter the results. The picture stimuli were derived from a set of pictures that were visually normed (Snodgrass \& Vanderwart, 1980), and the picture stimuli all had very high visual name agreement and recall scores. The blind individuals did not outperform the blindfolded sighted participants, a result that appeared to be inconsistent with an earlier report (Heller, 1989). However, Experiment 4 did not test for picture recognition but focused on spatial memory in a pictorial array. The numerically higher performance of the blind individuals means that it is very unlikely that increased sample sizes would yield higher performance by sighted participants.

It is important that there is a great deal of variability in the performance levels that can be seen in blind participants. Some are slow and not very accurate. This was the case for one person whose blindness was caused by diabetes, and for another who was older and very recently blinded. There were 
Table 5 Mean numbers correct for exchange and $45^{\circ}$ rotations in Experiment 4, mean study times in seconds, and mean response times in seconds, with standard deviations $(S D)$ in parentheses, for a picture change task with blind and blindfolded sighted participants

\begin{tabular}{llll}
\hline Group & No. Correct (Out of 12) & Exploration/ Study Time & Response Time \\
\hline Exchange Position & & & $227.6(99.8)$ \\
Blind & $8.7(2.0)$ & $297.5(69.6)$ & $195.4(64.3)$ \\
Blindfolded sighted & $8.1(2.8)$ & $345.6(28.5)$ & $244.3(92.7)$ \\
45 Rotation & & & $292.1(105.2)$ \\
Blind & $8.3(1.8)$ & $345.9(28.3)$ & $209.6(82.0)$ \\
Blindfolded sighted & $7.9(1.3)$ & & \\
\hline
\end{tabular}

Mean age of blind participants $=44$ years old, age range $=22-69$. Mean age of the blindfolded sighted participants $=42$ years old, age range $=19-62$. Maximum study time $=6 \mathrm{~min}$.

also very skilled blind individuals, with scores of 11 and 12 correct in the exchange position condition. Similarly, there were two sighted participants with scores of 11 and 12 in the exchange position condition. Blind individuals, like sighted persons, are extremely variable in their haptic skills.

\section{Experiment 5: Shape versus orientation change with letters}

This experiment was conducted in order to evaluate the role of stimulus familiarity on task difficulty in the haptic change task. Raised-line pictures were identified at a rather low level in Experiment 1 and in the controls in Experiment 3. Thus, it might prove difficult to notice orientation shifts from a canonical orientation when participants could not identify most of the stimuli. Also, some pictures of objects may generally appear in a variety of orientations - for example, an axe or umbrella. Whereas letters can be identified when tilted, they are generally viewed while reading with the stimuli at an upright, or close to upright orientation. Relatively simple and familiar configurations were used, namely print capital letters, to further examine the possible role of the lack of familiarity of the raised-line picture patterns for touch.

Participants felt a set of 12 raised-line print capital letters in the same relative locations in a smaller $21.6 \times 28 \mathrm{~cm}$ array, as in the earlier experiments reported here. Subsequently, they were asked to indicate which patterns were changed in a second set of stimuli, in which six of the letters were changed to geometric shapes. This geometrical shape change group was expected to perform better than two other groups with the same letters, but with orientation changes of $45^{\circ}$ or $90^{\circ}$. Because letters are defined by orientation and are generally upright, performance in rotation change groups was expected to be somewhat higher than in Experiments 1 and 2. Given the results of Experiment 2, the $90^{\circ}$ change group was expected to yield higher change task performance than the $45^{\circ}$ group.
Experiments that have demonstrated change blindness in touch (e.g., Yoshida et al., 2015) have typically removed any possibility of participants identifying the tangible stimuli. Their research paradigms used line orientation or spatial position as the change with simple straight, line-shaped vibrotactile configurations. In the present experiment we examined the additional impact of identity information on change detection by including a group with changes to different patterns. If identity information is an important component of the ability to notice changes, then this group should also be expected to outperform the groups involving orientation shifts from the upright. This initial experiment with print letters was expected to yield high performance, as it should be easy to detect categorical changes and any changes from a familiar, canonical orientation. Experiments 6 and 7 were designed to further isolate components of task difficulty.

\section{Method}

Participants There were 36 undergraduate volunteers in the experiment, with 12 in each of the three groups (age range = 18-27 years, with two participants over 21). The geometrical shape change group had ten females and two males, whereas the other two groups comprised 11 females and one male. None of the participants had served in Experiments 1-4.

Stimuli The letter stimuli in the standard study array were 12 print capital letters, all approximately $3 \mathrm{~cm}$ high. The letters (see Figs. 7-9). were smaller than the pictures in Experiments 1-3 (between 3.2 and $7 \mathrm{~cm}$ tall, with an 8.3-cm-long baseball bat). The raised-line capital letters (N, U, F, H, M, R, T, B, L, $\mathrm{G}, \mathrm{P}$, and J) were produced with a Swedish raised-line drawing kit that produces durable lines when a ball point pen is drawn over the plastic sleeve on a rubber board (see Heller, 1989). The letter size was large enough to permit ease of identification, as they were more than $1 \mathrm{~cm}$ in height (see Loomis, 1981). 
In the orientation change groups, the changed stimuli were rotated $45^{\circ}$ or $90^{\circ}$. All of the standard stimuli and changes in the three groups included stimuli in the same relative spatial locations as in the earlier experiments; the letters were upright in the initial standard stimulus array. They were produced on 8.5 in. $(21.6 \mathrm{~cm}) \times 11$ in. $(28 \mathrm{~cm})$ plastic sheets mounted on mat board. In one group, six of the 12 raised print letters were changed to geometric forms (see Fig. 8), in the same locations as the other changes in Experiments 1 and 2 (horizontal rhombus, parallelogram, square, circle, triangle, and hexagon). All of the stimuli were drawn with the aid of templates, and the stimulus sheets were placed on a foam board base that was designed to keep them at a constant orientation with respect to the participants.

Design and procedure The experiment had a between-group design, with independent groups for geometric shape change, $45^{\circ}$ rotation change, and $90^{\circ}$ rotation change. In most respects, the method was like that of Experiment 2, and the groups were simply told that there could be a change. As in the earlier haptic experiments, the participants were blindfolded throughout. Participants were told that the task was to feel the patterns for a full $6 \mathrm{~min}$, and learn "what and where they are." In addition, they were given unlimited time to explore the second set of patterns that included changes, but were told to try to aim for accuracy. The study period was reduced to $6 \mathrm{~min}$, since it was thought that this would be sufficient for haptic exploration, especially given the results of Experiment 4 . There, performance with $6 \mathrm{~min}$ had been similar to that for a longer study time in earlier experiments. After $6 \mathrm{~min}$, a second stimulus array was substituted for the first, standard sheet. The participants were instructed to feel the second set of stimuli and to indicate which ones were changed, in any way, by placement of small squares on those patterns that were changed. Feedback on performance was not given. Participants were not told that the letters were all upright in the study array. Moreover, they were not told that the standard stimuli were letters.
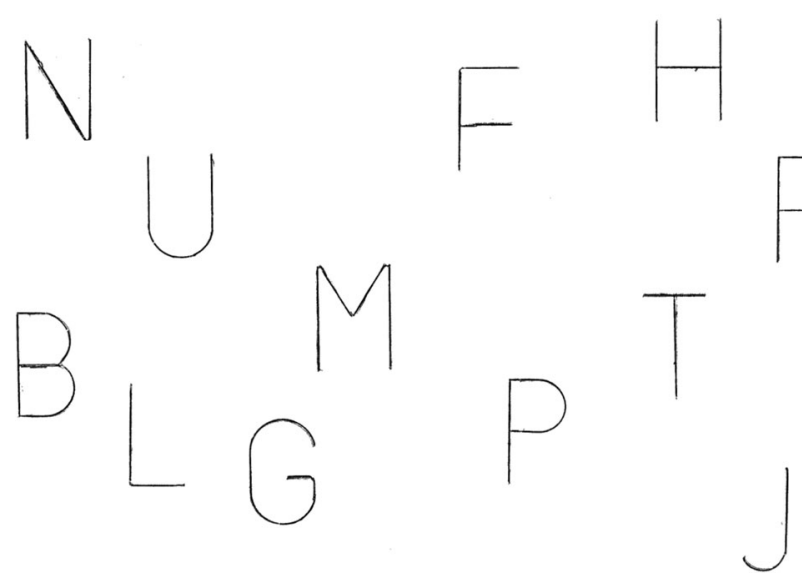

Fig. 7 The standard study array of raised-line letters.

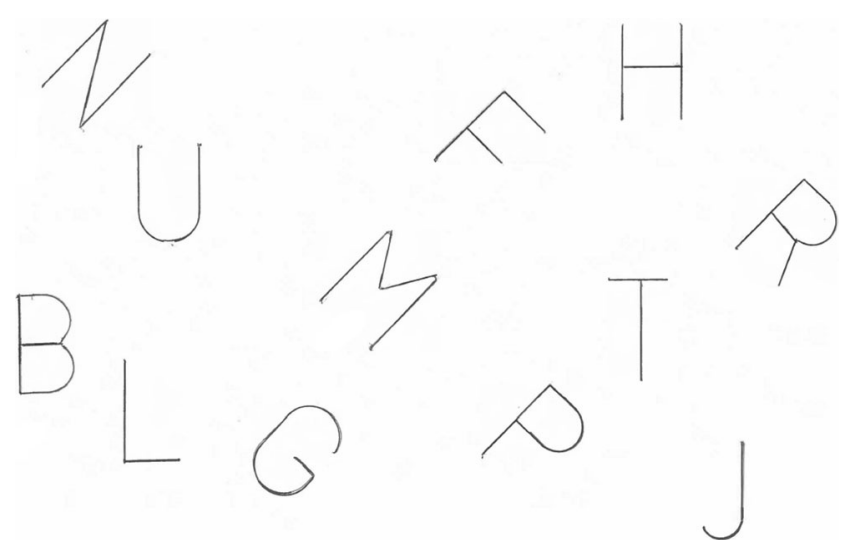

Fig. 9 The $45^{\circ}$ rotation change stimulus array. The same letters were turned for the $90^{\circ}$ change test array.

\section{Results and discussion}

Performance was high in the group that was first presented with print letters and then had to indicate the changes involving geometrical shapes $(M$ correct $=11.1, M \%$ correct $=92.5)$. Lower performance was obtained with $90^{\circ}$ rotation changes $(M=9.9, \%$ correct $=82.5)$ and $45^{\circ}$ rotation changes $(M=$ $8.92, \%$ correct $=74.3$; see Table 6 ). An ANOVA on numbers correct indicated the effect of type of change was significant, $F(2,33)=8.2, p=.0013, \eta_{\mathrm{p}}{ }^{2}=.25$. However, a Tukey HSD test indicated that whereas the geometrical shape change group mean differed significantly from that in the $45^{\circ}$ change group $(p=.01)$, the other comparisons failed to reach significance.

A second ANOVA on response latencies showed the effect of group was nonsignificant, $F(2,33)=0.60, p=.55$. Response times were similar for the shape change $(M=$ $224.4 \mathrm{~s}), 45^{\circ}$ change group $(M=252.2 \mathrm{~s})$, and $90^{\circ}$ change ( $M=223.0 \mathrm{~s})$ groups.

The haptic change task was very easy when the change consisted of a categorical shift from letters to shapes. Performance was high, in terms of accuracy, and it is likely that it was at or close to ceiling. This outcome is consistent
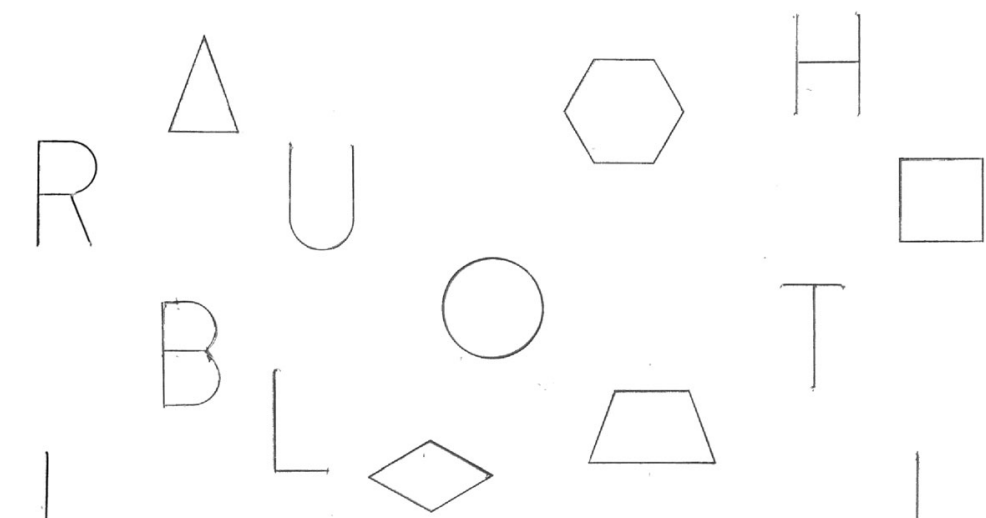

Fig. 8 The change stimuli using geometric shapes. 
with an explanation in terms of familiarity of the stimuli. It was easier to notice changes for print letter stimuli that are easy to identify and name. Note that letters are normally experienced in an upright orientation and are defined by orientation. Deviations from this familiar canonical orientation were noticeable, even with smaller stimuli than in Experiments 1 and 2.

One could readily argue that the changes from canonical orientations simply required that participants notice a change from the initial display. In fact, this is a possible interpretation, but good performance also requires that the participants know that the items during study were all print letters and were all initially upright. Thus, if participants noticed that the original stimuli were all letters, it would be easy to pick out changed stimuli consisting of familiar geometrical shapes. Also, the participants could respond correctly if they noticed that the new stimuli were all closed forms without internal lines. They would have had to realize that the original stimuli did not have either of these characteristics. Note that the orientation changes were from a familiar upright canonical orientation. This should aid participants, if they noticed and recalled that the original stimuli were all upright. Thus, it might not be possible to completely separate the effect of noticing change from a familiar orientation from detailed recall of the orientation of each item in the initial display. This would require additional experimentation. Future research should probably involve letters that are initially at various orientations to provide further clarification of the role of canonical orientations (see Exp. 7).

\section{Experiment 6: Letter exchange compared with changes involving new letters}

Performance in the change task in Experiment 5 was high when the stimuli were familiar letter patterns and the change involved the category of half of the letters changing to geometric shapes. Also, performance was good with $90^{\circ}$ rotations. This higher performance with letters can be ascribed to a number of factors, but one likely explanation involves pattern familiarity and knowledge of pattern identity. Additional support for this interpretation could be found, if scores were higher in the change task when new stimuli were substituted for old ones, as compared with just exchanging the locations of stimuli. The exchange manipulation forces individuals to remember the identity and location of the stimuli. If the change merely involves identity, correct responses could occur without retention of correct location information. This should be easier with familiar, identifiable stimuli, and this task should place less of a load on memory. Consequently, Experiment 6 provided a direct comparison of a haptic letter exchange position group with one in which new letters were substituted for old ones in the change task. This experiment was also intended to further test the extreme idea that when using touch, one has little or no understanding of the spatial relations in a complex display (e.g., Yoshida et al., 2015). It is important that this earlier report by Yoshida et al. used patterns that were all identical, namely vibrotactile lines that varied in location or orientation. The present experiment was intended to examine the role of pattern identity in the change task, since the lack of any difference between patterns, other than location or orientation, might help explain the low levels of performance found in haptic conditions in Yoshida et al.

\section{Method}

Participants There were 12 female participants in the new letter change group. An additional 12 females served in the group in which letter locations were exchanged (total $N=24$ ). None had served in the prior experiments, and they ranged in age from 18-41 (three were over 22).

Stimuli The stimuli were raised-line print capital letters, as in Experiment 5. The exchange position group used the same letters as in Experiment 5, with the change involving exchange of three letters with three others. In the new-letter group, the same raised-line print capital letters were used for the standard study presentation. In the new-letter change array, the $\mathrm{N}$ was replaced by an $\mathrm{X}$, the $\mathrm{M}$ was replaced by a $V$, the $F$ was replaced by an $A$, the $R$ was replaced by an $I$, the $P$ was replaced by an $S$, and the $G$ was replaced by a $\mathrm{D}$. The new letters were randomly selected from the alphabet, with the restriction that letters did not repeat.

Table 6 Mean numbers correct for the haptic change task in Experiment 5, mean solution times (in seconds), and letter identifications for print capital letter standard stimuli (with $S D$ s in parentheses)

\begin{tabular}{llll}
\hline Group & Correct Change & Time Score (Seconds) & Letter Identification \\
\hline Shape change & $11.1(.9)$ & $224.4(98.3)$ & 11.0 \\
$45^{\circ}$ change & $8.9(1.2)$ & $252.2(58.6)$ & 11.3 \\
$90^{\circ}$ change & $9.9(1.7)$ & $223.0(55.9)$ & 11.5 \\
\hline
\end{tabular}

Blindfolded participants in Experiment 5 were told to learn "what and where the patterns are." They were not told about the nature of the change. Maximum score possible $=12$ for change detection and letter identification. Maximum study time $=6$ min. 
Design and procedure The experiment had a simple betweengroup design, with participants being exposed to changes involving exchange or the substitution of new letters. As in Experiment 3, all participants were told they needed to learn what and where the patterns were on the array during study. They were not told what sort of patterns to expect. The exposure time was $6 \mathrm{~min}$, with unlimited time for responses. The participants were instructed to take as much time as needed for accuracy.

After the completion of the change task, the participants were asked to feel the stimuli in the second stimulus sheet and to identify the patterns. They were also asked whether they knew what type of patterns were involved. Almost all of the participants were aware that the initial study stimuli were all letters.

\section{Results and discussion}

Change detection performance was higher for the new-letter group ( $M=10.4$ correct, $S D=1.6)$ than for the exchange position group $(M=8.6$ correct). This advantage for the new-letter group was significant, $t(22)=2.25, p=.035$ (see Table 7). However, the effect of group did not reach significance for response times, $t<1$. Note that subsequent letter identification was very high and similar for the new-letter group $(M$ correct $=11.2)$ and the exchange position group $(M$ correct $=11.0), t(22)=0.32, p=.75$.

There are a number of possible explanations for relatively high performance when using letters in the haptic change task. Letters are familiar configurations, and familiarity may aid memory. Change task performance is improved when it is easy to identify patterns. Also, letter shapes permit recall using auditory representations and phonemic encoding. Indeed, it is likely that participants engage in dual coding (see Paivio, 1965), and represent the patterns in the form of a verbal phonemic code as well as visual representations. Visual imagery is known to aid recall, and blindfolded sighted participants are likely to visually recode haptic patterns (e.g., Lederman, Klatzky, Chataway \& Summers, 1990; Revesz, 1950). These explanations will be taken up again in the General Discussion.

Another possible explanation of the high level of performance ( $M=87 \%$ correct) for the new-letter group should be considered. The instructions were to "learn what and where the patterns are" on the array. This differed from the earlier instructions in Experiments 1 and 2. It is possible that the instruction to learn what the stimuli were aided participants. Lack of clarity in the instructions can hinder performance in the change task. Consequently, an additional 12 participants (two males; age range $=18-22$ years) were exposed to the new-letter change task, but they were simply told that some of the stimuli could be changed and they needed to indicate which ones were changed on the test portion of the protocol. Otherwise, the method was like that of Experiments 2 and 5.
No information was given about the nature of the patterns. The participants were asked to feel and vocally name the patterns after completion of the change task on the changed array, but the were not told about this in advance. When participants were just told that patterns could be "changed," the mean number correct $(M=10.1, S D=2.0)$ was nearly identical to that for the participants who were told to learn "what and where" the stimuli were $(M=10.4)$ in Experiment 6 . The difference between the accuracy means was nonsignificant, $t$ $<1$, as was the effect on response time, $t<1$. These results suggest that when participants are told to expect a change, they may spontaneously take this to mean a change in the identity or location of a pattern. Perhaps they may be less likely to anticipate orientation shifts.

The change task is clearly easier when new letters are substituted for older ones. A correct response with new letters merely requires that participants recall the earlier set of letters. However, the task is more complicated and places a greater load on memory in exchange conditions. Here, the participants need to recall item information, along with the spatial locations of the letters in the original array. The importance of item information in the change task may help explain the very low levels of performance reported by Yoshida et al. (2015), as all of their tactile stimuli were vibrotactile straight lines that varied in orientation and/or location.

Of course, possible additional factors are at play and the comparison between item substitution versus location exchange is complex, and caution is warranted when considering the results. New letters can vary in similarity to those that they replace, and location changes may be influenced by the spatial context, as well as spatial similarity. Thus, the same confusions might arise in both of the senses of vision and touch.

\section{Experiment 7: Participants told letters would be turned or moved}

This experiment was similar to Experiment 1, with a couple of differences. Print capital letters were used, as in Experiments 5 and 6 . The participants were told that they would be feeling a number of print capital letters and that they had to learn what and where the letters were. In the $45^{\circ}$ and $90^{\circ}$ groups, they were instructed that the task was to indicate which letters were turned, whereas the exchange position participants were told that some of the letters were moved to new locations. Performance was expected to be higher for the change task including letters in the present experiment, than in the earlier, similar haptic experiments that included pictures. This was the case, since letters generally appear in an upright orientation, or close to an upright orientation, and deviations from the canonical orientation should be most obvious. Explicit information about the nature of the changes was expected to aid 
Table 7 Mean numbers correct in Experiment 6 for the haptic letter exchange task versus new letter changes, with mean solution times (with SDs in parentheses) and mean correct identifications

\begin{tabular}{llll}
\hline Group & Correct Change & Time Score (Sec) & Letter Identification \\
\hline Exchange position & $8.6(2.3)$ & $252.4(103.2)$ & $11.0(1.5)$ \\
Change new letters & $10.4(1.6)$ & $237.5(91.5)$ & $11.2(1.0)$ \\
Change new letters, only told "changed" & $10.1(2.0)$ & $276.4(108.9)$ & $11.4(.8)$ \\
\hline
\end{tabular}

Maximum score possible $=12$ for change correct and letter identification.

performance and yield optimal performance levels. Again, the aim was to examine the extreme idea that when using touch, participants lack the ability to remember spatial displays (Yoshida et al., 2015).

\section{Method}

Participants There were 12 individuals in each of three groups: exchange (four males), $45^{\circ}$ rotation change (two males), and $90^{\circ}$ rotation change (one male). The age range was 19-28 years. As in earlier experiments, most of the participants were under 22 years of age. None had served in any of the earlier experiments reported here.

Stimuli The stimuli were raised-line print capital letters, as in Experiments 5 and 6. The letter stimuli in the exchange position group were identical to those in earlier experiments, as were the rotation stimuli.

Design and procedure Experiment 7 had a simple betweengroup design, with the groups comprising exchange position and $45^{\circ}$ and $90^{\circ}$ rotation changes. Blindfolded participants were told in advance that they would feel print capital letters, and the exchange position group were instructed that some letters would be moved to new locations. The rotation change participants were told that some letters would be turned from their original orientation and that they were to indicate which ones were turned with the placement of squares. They were not told that the letters were originally upright, nor were they given any feedback about their responses. In some other respects, the procedure was similar to that of Experiment 1, but the stimuli were print capital letters. As in Experiments 5 and 6 , the study time was 6 min.

After completion of the change task, the participants were asked to identify the letters using touch. Those in the two rotation change groups were reminded that some of the letters were turned and that they should name the letter prior to rotation.

\section{Results and discussion}

Performance was high across all groups: exchange position $(M=9.8,81.7 \%$ correct $)$, and particularly in the $45^{\circ}(M=$
$11.0,91.7 \%$ correct $)$ and $90^{\circ}(M=11.3,94.2 \%$ correct; see Table 8 ) groups. Despite the high accuracy across groups, a one-way between-group ANOVA showed that the effect of group was significant, with higher change task performance in the two rotation change groups, $F(2,33)=4.1, p=.025, \eta_{\mathrm{p}}{ }^{2}$ $=.167$. Change task performance was over $90 \%$ correct for the rotation groups, but somewhat lower, at $81.7 \%$ correct, in the exchange position group. Tukey HSD tests showed that the $45^{\circ}$ and $90^{\circ}$ rotation group means did not differ from each other, but both were significantly different from the exchange position group mean, $p<.05$. The effect of group on response latency was nonsignificant, $F(2,33)=1.15, p=.33$.

Rotation changes are not very difficult to detect when participants expect them and the stimuli are letters. Performance was likely at or near ceiling in the orientation groups. Even though smaller than hand size, letters are easy to discriminate and identify. This likely contributed to high performance in the change task. Some participants demonstrated varying strategies in an attempt to determine whether individual letters were upright. A few scanned the outside border of the foam board framework, using the side edges as a reference point when feeling a tilted letter. Thus, a participant felt the side of the oblique " $\mathrm{R}$ " and then felt the nearby vertical edge of the framework before making a judgment. This provided her with a vertical frame of reference to guide her judgments. Another participant used an egocentric scanning method before judging the orientation of other letters. She moved her index finger back and forth, toward and away from her body, at the midline. Subsequently, she felt the letter $\mathrm{G}$ before deciding whether the letter was turned at an oblique orientation. It is likely that the instruction that some of the letters would be turned helped prompt these exploration methods.

One would expect lower performance if the initial presentation involved letters at orientations that varied from the upright, and this will be considered more completely in future investigations. Conceivably, correct responses in the present experiment could have derived from participants noticing any change from the familiar, upright canonical orientation. Consequently, we tested four participants with $45^{\circ}$ rotations using touch, but we reversed the order of presentation. The test standard was presented first, with half of the letters turned and half upright (see Exp. 2, visual tests with pictures in reversed testing order). The participants were told that they 
Table 8 Mean numbers correct for the haptic change task in Experiment 7, mean solution times (in seconds), and mean correct identifications, for print capital letter standard stimuli (with $S D$ s in parentheses)

\begin{tabular}{llll}
\hline Group & Correct Change & Time Score (Seconds) & Letter Identification \\
\hline Exchange position & $9.8(1.7)$ & $255.2(105.1)$ & 11.8 \\
$45^{\circ}$ change & $11.0(1.5)$ & $286.2(63.2)$ & 11.6 \\
$90^{\circ}$ change & $11.3(0.9)$ & $313.1(106.1)$ & 11.7 \\
\hline
\end{tabular}

Participants were told the letters could be moved or turned, as appropriate. Participants were told to learn "what and where the patterns are." Maximum score possible $=12$ for change detection and letter identification. Study Time $=6 \mathrm{~min}$.

should indicate which stimuli were turned from their original positions on the display. On testing, all of the letters were upright. The mean number correct was $8.75(M=72.9 \%)$, and this was lower than the very high performance obtained in the results of Experiment $7(M=91.7 \%)$.

\section{General discussion}

The present experiments yielded several important results. Haptic memory for spatial layout clearly depends on stimulus familiarity and the nature of the stimuli that are used. Tangible pictures are complex arrays, and when small, are difficult to identify, recall, and localize in larger arrays. Participants showed change task performance gains when they knew the identity of the pictures in Experiment 3. They also performed at a higher level when the stimuli were more familiar - raisedline capital letters. There are many possible explanations of these performance differences between pictures and letters, and they will be considered in the discussion that follows.

Instructions matter, as a $45^{\circ}$ rotation was difficult to notice in the haptic picture change task, when no information was given about the nature of the changes in the stimulus array in Experiment 2. However, large orientation shifts were salient, as shown by better performance given $90^{\circ}$ rotations. Overall, changes in location were not any easier to cope with than large orientation shifts, but $45^{\circ}$ rotations were more difficult in Experiment 2, and this is consistent with the presence of an oblique effect in vision and touch (Appelle, 1972; Gentaz et al., 2008). Note that performance was much higher for rotations when the stimuli were letters and the instructions were explicit regarding the type of change (moved or turned) in the final experiment. Performance was near or at ceiling, even for $45^{\circ}$ rotations.

Letters are familiar forms, and familiarity can aid retention. The consequences of this are manifold. It is more difficult to remember the locations of a set of patterns if one does not know what they are. First, participants may engage in multisensory encoding of the tangible letters and they can encode them in terms of subvocal phonemic codes. They may also encode them as movement patterns. In addition, it is likely that visual imagery is triggered by haptic exploration of the letters, and this can also aid recall. Also, letters are highly discriminable. Finally, the possible set of letters is limited, whereas tangible pictures can be innumerable objects or can even be abstract. Consequently, it is more difficult to remember the locations of a set of patterns such as unnamed pictures, albeit familiar once seen, if one does not know what they are.

Note that some tangible pictures are easy to recognize and identify, even without any assistance (Kalia \& Sinha, 2011). It is clear that many raised-line letters are readily identifiable, if large enough, but why is this the case? Perhaps, when one feels a letter, the haptic exploration and movement patterns resemble the movements that one engages in when printing the letter with a pen or pencil. This may facilitate recognition, if these motion patterns are stored as representations of the letters. It is suggested that haptic exploration of a raised-line heart prompts movement patterns that are similar to those one would use to draw it, as with letters. Children are experienced in drawing simple configurations, like a heart and moon. Perhaps this motoric code can contribute to the very high recognition levels reported for some tangible pictures.

The results suggest that it may be easier to notice changes in the identity of patterns than in some of their spatial attributes, especially location. This is the case for letters, for which the patterns are familiar and identification is easy. It clearly helps performance in detecting the changes in an array when participants know what the patterns represent, for example naming pictures during study aided performance in the change task in Experiment 3. Haptic picture exchange performance was high $(M=9.6)$ for participants given knowledge of picture identity. Knowledge of the identity of a pattern may facilitate the generation of an image, and imagery may aid memory.

An important factor in letter change detection may be that letters are defined by their spatial orientation. Thus, a $90^{\circ}$ rotation of an $\mathrm{N}$ changes letter identity. Changes in letter orientation also represent deviations from canonical orientations. People can certainly read printed material that is tilted, but the present experiments involved individual patterns in isolation and without a semantic context. However, many pictures can appear in a variety of orientations, and this would not be unusual. For example, a bear can walk up or down a $45^{\circ}$ slope 
on a mountain, but not generally descend head first down a $90^{\circ}$ slope. Flags can be viewed as upright or at a slant. An umbrella can be held at a variety of angles, as in the wind. Deviations from familiar, canonical orientations are more readily detected when those deviations are large (e.g., 90 ${ }^{\circ}$ ) or for patterns that are defined by their upright orientation, namely letters. Haptic letter exploration takes less time than haptic exploration of pictures, and accurate identification is much higher. The use of more familiar patterns may have allowed participants relatively more time to study the spatial layout, since they would need less time for pattern identification. Perhaps participants spontaneously interpret "change" to imply a change in item identity, unless instructed otherwise. They may then attempt to identify the pictures that they feel, even if not asked to name them during study. This interpretation is supported by the results of Experiment 6. Here there was no difference between detecting change in letter identity, whether or not the instructions referred to letters "changed" or to study "what and where the patterns" were.

The high level of performance in the experiments involving letters likely derived from the combined effects of knowledge of shifts from the canonical orientation in combination with recall of individual patterns. Future research will enable the separation of these two processes. Nonetheless, it is telling that changes from a familiar orientation can be easy to detect. This provides further support for the idea that item familiarity matters in the change task. It is also important to mention that some of the spatial manipulations in the experiments reported here involve changes that are not entirely comparable. The change to a different form, for example, is not exactly the same as an orientation shift. Nonetheless, the methods allowed an examination of task difficulty for a variety of spatial changes. This allowed the work to shed light on some of the sources of difficulty in the haptic change task.

The outcome indicated that the previously reported generalized advantages of females in the change task may not be reliable (Heller et al., 2010), are probably dependent upon task requirements and consequently are not generalizable across contexts. In Experiment 1 we used much larger numbers of participants than in the earlier study. If females hold any advantages over males in haptic memory for spatial arrays, large individual differences often outweigh any of these possible advantages.

The present results have clear implications for the education and rehabilitation of people with visual impairments. Landmark locations and orientation of lines within a layout are important in the interpretation of tangible maps, and these maps often include diagonal lines. The results of the present experiments are clearly relevant to rehabilitation and educational strategies for instruction in map use and other graphics for blind individuals. It is suggested that instruction in map use should include information about the upright orientation and the importance of keeping this constant. This recommendation is relevant to posture and the spatial array. Also, prior information about symbol identity is likely to be helpful.

To summarize, the results of experiments on the change task suggest that a lack of familiarity with particular types of configurations in haptics may contribute to task difficulty, especially with small tangible pictures in a spatial array. Knowledge of picture identity boosts performance in the haptic change task. Haptic change task performance is facilitated when familiar tangible letters are used and pattern identity is known. Oblique orientations are potentially problematic for touch, but only when no information is provided about the change that may be expected.

Acknowledgments Experiment 4 was supported by a PIF grant by Eastern Illinois University to M.H. We are grateful for the participation of the blind participants in Experiment 4, and to Joseph Morger for assistance with data collection in Experiment 4.

\section{References}

Appelle, S. (1972). Perception and discrimination as a function of stimulus orientation: The "oblique effect" in man and animals. Psychological Bulletin, 78, 266-278. https://doi.org/10.1037/ h0033117

Auvrey, M., Gallace, A., Tan, H. Z., \& Spence, C. (2007). Crossmodal change blindness between vision and touch. Acta Psychologica, 126, 79-97.

Eals, M., \& Silverman, I. (1994). The hunter-gatherer theory of spatial sex differences: Proximate factors mediating the female advantage in recall of object arrays. Ethology and Sociobiology, 15, 95-105.

Gallace, A., Tan, H. Z., \& Spence, C. (2006). The failure to detect tactile change: A tactile analogue of visual change blindness. Psychonomic Bulletin \& Review, 13, 300-303. https://doi.org/10.3758/ BF03193847

Gentaz, E., Baud-Bovy, G., \& Luyat, M. (2008). The haptic perception of spatial orientation: A review. Experimental Brain Research, 187, $331-348$

Gentaz, E., \& Hatwell, Y. (1995). The haptic “oblique effect" in children's and adults' perception of orientation. Perception, 24, 631-646.

Heller, M. A. (1989). Picture and pattern perception in the sighted and blind: The advantage of the late blind. Perception, 18, 379-389.

Heller, M. A., Calcaterra, J., Green, S., \& Lima, F. (1999). The effect of orientation on braille recognition in persons who are sighted and blind. Journal of Visual Impairment and Blindness, 93, 416-419.

Heller, M. A., Jones, M., Walk, A. M., Schnarr, R. E., Hasara, A. N., \& Litwiller, B. (2010). Sex differences in the haptic change task. Journal of General Psychology, 137, 49-62.

James, T., \& Kimura, D. (1997). Sex differences in remembering the locations of objects in an array: Location-shifts versus location-exchanges. Evolution and Human Behavior 18, 155-163.

Kalia, A., Hopkins, R., Jin, D., Yazzolino, L, Verma, S., Merabet, L., \& Sinha, P. (2014). Perception of tactile graphics: Embossings versus cutouts. Multisensory Research, 27, 111-125.

Kalia, A., \& Sinha, P. (2011). Tactile picture recognition: Errors are in shape acquisition or object matching? Seeing and Perceiving, 25, 287-302.

Kennedy, J. M., \& Bai, J. (2002). Fit judgments predict identification, recognition memory, and confidence. Perception, 31, 1013-1026. 
Lederman, S. J., Klatzky, R. L., Chataway, C., \& Summers, C. D. (1990). Visual mediation and the haptic recognition of two-dimensional pictures of common objects. Perception \& Psychophysics, 47, 54-64.

Linn, P.-H, \& Luck, S. J. (2012). Proactive interference does not meaningfully distort visual working memory capacity in the canonical change detection task. Frontiers in Psychology, 3, 1-9.

Loomis, J. M. (1981). On the tangibility of letters and Braille. Perception \& Psychophysics, 29, 37-46.

Luck, S. J. (2008). Visual short-term memory. Scholarpedia, 2(6), 3328. https://doi.org/10.4249/scholarpedia.3328

Millar, S. (1994). Understanding and representing space: Theory and evidence from studies with blind and sighted children. Oxford, UK: Oxford University Press.

Millar, S. (1997). Reading by touch. London, UK: Routledge.

Paivio, A. (1965). Abstractness, imagery, and meaningfulness in pairedassociate learning. Journal of Verbal Learning and Verbal Behavior, 4, 32-38.

Pashler, H. (1988). Familiarity and visual change detection. Perception \& Psychophysics, 44, 369-378. https://doi.org/10.3758/BF03210419

Postma, A., Izendoorn, R., \& De Haan, E. H. F. (1998). Sex differences in object location memory. Brain and Cognition, 36, 334-345.

Revesz, G. (1950). Psychology and art of the blind. London, UK: Longmans Green.

Silverman, I., \& Eals, M. (1992). Sex differences in spatial abilities: Evolutionary theory and data. The adapted mind: Evolutionary psychology and the generation of culture. In J. Barkow, L. Cosmodes, \& J. Tooby (Eds.). New York, NY: Oxford University Press, pp. 533549.
Simons, D. J. (2000). Current approaches to change blindness. Visual Cognition, 7, 1-15. https://doi.org/10.1080/135062800394658

Snodgrass, J. G., \& Vanderwart, M. (1980). A standardized set of 260 pictures: Norms for name agreement, image agreement, familiarity, and visual complexity. Journal of Experimental Psychology: Human Learning and Memory, 6, 174-215. https://doi.org/10. 1037/0278-7393.6.2.174

Voyer, D., Nolan, C., \& Voyer, S. (2000). The relation between experience and spatial performance in men and women. Sex Roles, 43, 891-915.

Voyer, D., Postma, A., Brake, B., \& Imperato-McGinley, J. (2007). Gender differences in object location memory: A meta-analysis. Psychonomic Bulletin \& Review, 14, 23-38. https://doi.org/10. 3758/BF03194024

Wijntjes, M. W., van Lienen, T., Verstijnen, I. M., \& Kappers, A. M. L. (2008). The influence of picture size on recognition and exploratory behavior in raised-line drawings. Perception, 37, 602-614.

Wood, K, \& Simons, D. J. (2017). Reconciling change blindness with long-term memory for objects. Attention, Perception, \& Psychophysics, 79, 438-448.

Yoshida, T., Yamaguchi, A., Tsutsui, H., \& Wake, T. (2015). Tactile search for change has less memory than visual search for change. Attention, Perception, \& Psychophysics, 77, 1200-1211. https://doi. org/10.3758/s13414-014-0829-6

Publisher's note Springer Nature remains neutral with regard to jurisdictional claims in published maps and institutional affiliations. 